\title{
Localization in Strongly Chaotic Systems
}

\author{
Mario Feingold \\ Dept. of Physics, Ben-Gurion University, Beer-Sheva 84105, Israel
}

\begin{abstract}
We show that, in the semiclassical limit and whenever the elements of the Hamiltonian matrix are random enough, the eigenvectors of strongly chaotic time-independent systems in ordered bases can on average be exponentially localized across the energy shell and decay faster than exponentially outside the energy shell. Typically however, matrix elements are strongly correlated leading to deviations from such behavior.
\end{abstract}

PACS numbers: 03.65.Sq, 05.45.+b

submitted to Phys. Rev. Lett., April 1996

The quantum mechanical behavior of strongly chaotic systems is commonly thought to be of two, apparently different types. On one hand, there are the time-periodic systems, e.g. the Kicked Rotor, for which the eigenstates of the one period evolution operator, $U$, are under certain conditions localized. ${ }^{1}$ When this occurs, there is no repulsion between most levels and the spectrum of quasienergies is characterized by a Poisson spacings distribution. $^{2}$ One the other hand, one has the timeindependent systems, e.g. the Coupled Quartic Oscillators, where eingenstates in a phase space representation, are on average homogeneously spread over the corresponding energy shell. Such states are being regarded as extended leading to strong level repulsion and a Wigner spacing distribution. ${ }^{3}$ It is the purpose of this Letter to show that as far as the behavior of the eigenstates and in particular their localization properties, the two types of systems are in fact quite similar and that localization can occur under certain conditions in time-independent systems as well.

For simplicity, in what follows we shall contain our discussion to a subclass of the time-periodic systems, namely, to kicked systems of the type

$$
H=K(p)+V(q) \sum_{n=-\infty}^{\infty} \delta(t-n T) .
$$

Such systems can be mapped onto Anderson models for the motion of electrons on a purely one-dimensional disordered lattice. Whenever the corresponding hopping elements, $W_{n}$, decay faster than $n^{-1}$ and the the site energies, $\epsilon_{n}$, are random enough, the resulting eigenstates of the Anderson model are known to be exponentially localized. A more direct approach is based on the fact that in the basis of eigenstates of $K(p)$ the evolution operator, $U_{n m}$, is a banded matrix with elements that have pseudo-random phases. Therefore, its eigenvectors are expected to behave similarly to those of a banded random matrix ${ }^{4}$ of the same band width, $b$. Since the latter can be analyzed using a transfer matrix formalism, the eigenvectors are exponentially localized with a localization length, $\xi$, that is proportional to $b^{2}, \xi=\gamma b^{2}$.

The Kicked Rotor for example, corresponding to $K(p)=p^{2} / 2$ and $V(q)=k \cos q$, was originally introduced as a simplified but representative Poincare map of a time-independent system with two degrees of freedom, $d=2$, also known as the Standard Map. It is therefore natural to expect that also its quantum mechanics should be reminiscent of that of time-independent systems. One obstacle that has prevented the search for this similarity is the fact that eigenstates of time-independent systems are best understood in phase space representations through the Berry-Voros conjecture. ${ }^{5}$ However, in order to obtain an analogous description to that leading to the banded evolution operator, $U_{n m}$, a basis with a natural ordering is required. Let, $H=H_{0}+V$, be an arbitrary separation of $H$. Using the eingenvectors of $H_{0}, v_{n}$, arranged in increasing order of the corresponding eigenvalues, $E_{0, n}$, one obtains for $H$ a matrix representation, $H_{n m}$, that is banded and moreover, has diagonal elements which vary on classical energy scales. Although the latter feature which is related to energy conservation is absent in the $U_{n m}$ matrix where diago- 
nal elements are on average of unit absolute value, we find that in the semiclassical limit, $\hbar \rightarrow 0$, the influence of the diagonal on the behavior of the eigenvectors is partially suppressed. As a consequence, in such bases, the semiclassical global structure of the $U_{n m}$ and $H_{n m}$ matrices is very much alike. On the other hand, a simple mechanism that generates randomness in the $U_{n m}$ matrix is absent in the case of $H_{n m}$, making the latter significantly less random.

We now turn to study the structure of the $H_{n m}$ matrix. Let us assume that all the relevant operators, namely, $H$, $H_{0}$ and $V$, are well behaved functions of the canonical variables, $\mathbf{z} \equiv(\mathbf{q}, \mathbf{p})$. Then, under quite general conditions, it is known ${ }^{6}$ that the energy average of the diagonal elements equals to the corresponding microcanonical average, that is,

$$
<H_{n n}>\simeq\{H(\mathbf{z})\}_{E_{0, n}}
$$

where for any function, $F(\mathbf{z})$,

$$
\{F(\mathbf{z})\}_{E_{0}} \equiv \frac{\int d \mathbf{z} F(\mathbf{z}) \delta\left[E_{0}-H_{0}(\mathbf{z})\right]}{\int d \mathbf{z} \delta\left[E_{0}-H_{0}(\mathbf{z}]\right)} .
$$

Moreover, the variance of the diagonal elements, $\sigma_{D}^{2} \equiv<H_{n n}^{2}>-<H_{n n}>^{2}$, was shown ${ }^{7}$ to be equal to twice the variance of the off-diagonal matrix elements that are close to the diagonal, $\sigma_{O}^{2}$, and correspondingly, $\sigma_{D}^{2}=O\left(\hbar^{d-1}\right)$. Therefore, in the semiclassical limit the distribution of diagonal elements has vanishing width whenever $d>1$. As we now show, this fact is extremely helpful in understanding the behavior of the off-diagonal matrix elements of $H_{n m}$. In the $n$-th row of $H_{n m}$, the average distance of matrix elements from the diagonal measured in units of energy is

$$
\begin{aligned}
\left(\Delta E_{0, n}\right)^{2} & \equiv \frac{\sum_{m}\left(E_{0, m}-E_{0, n}\right)^{2}\left|H_{n m}\right|^{2}}{\sum_{m(\neq n)}\left|H_{n m}\right|^{2}} \\
& =\frac{\left(\left[H_{0}, H\right]^{2}\right)_{n n}}{\left(H^{2}\right)_{n n}-(H)_{n n}^{2}},
\end{aligned}
$$

and using Eq. (2)

$$
<\left(\Delta E_{0, n}\right)^{2}>\rightarrow \hbar^{2} \frac{\left\{\left[H_{0}, H\right]_{P B}^{2}\right\}}{\left\{H^{2}\right\}-\{H\}^{2}} \quad \text { for } \hbar \rightarrow 0
$$

where the commutator of Eq. (4) was replaced by $\hbar$ times the corresponding Poisson bracket, $[\ldots]_{P B}$. Notice that while Eq. (2) holds for arbitrary values of $\hbar$, Eq. (5) only applies for small enough $\hbar$. The reason is that in Eq. (5), the lhs is the average of a ratio of diagonal elements which, in general, differs from the ratio of the averages on the rhs. However, if $d>1$ and $\hbar \rightarrow 0$, then the width of the distribution of the diagonal elements appearing in the denominator of Eq. (4) becomes vanishingly small, therefore playing the role of a constant in the averaging.

In order to fully grasp the implication of Eq. (5), it is necessary to express $<\left(\Delta E_{0, n}\right)^{2}>^{1 / 2}$ in terms of the number of states by multiplying it with the mean density of states, $\rho\left(E_{0}\right)$, which is $O\left(\hbar^{-d}\right)$, such that $<\left(\Delta N_{0, n}\right)^{2}>^{1 / 2}=O\left(\hbar^{1-d}\right)$. On one hand, for $d>1$ and $\hbar \rightarrow 0,<\left(\Delta N_{0, n}\right)^{2}>^{1 / 2}$ diverges. On the other hand however, the number of states in any classical energy range which, in turn, corresponds to the size of the truncated Hamiltonian matrix, $N$, is $O\left(\hbar^{-d}\right)$ and therefore diverges much faster. In fact, $<\left(\Delta N_{0, n}\right)^{2}>^{1 / 2} / N=O(\hbar)$ and accordingly, for small enough $\hbar$, the $H_{n m}$ matrix is banded. Moreover, Eq. (2) implies that the diagonal matrix elements, $H_{n n}$, grow on average as the volume of the energy shell grows. Both these features are absent in the traditional Random Matrix Ensembles, e.g. GOE, and this lack of structure leads to extended eigenvectors. A Random Matrix model which does include a simplified version of this structure is the Wigner ensemble ${ }^{8,9}$ which is composed of banded matrices of band width, $b$, with diagonal matrix elements that increase on average with constant rate, $\alpha$. Specifically, $\left\langle h_{n m}>_{e}=\alpha n \delta_{n m}\right.$, where $<\ldots>_{e}$ denotes ensemble averaging, and $\sigma_{n m}^{2} \equiv<h_{n m}^{2}>_{e}-<h_{n m}>_{e}^{2}=$ $1+\delta_{n m}$ for $|n-m|<b$ and vanishes otherwise. As in the case of the GOE, it is assumed that the strongly chaotic nature of the classical dynamics is sufficient to ensure that the matrix elements are uncorrelated random variables. In what follows we shall use the Wigner ensemble and its properties in order to understand the behavior of the eigenvectors of $H_{n m}$.

For $\alpha=0$ the Wigner ensemble is equivalent to the Banded Random Matrix Ensemble (BRME) which in turn can be thought of as an Anderson model with random, long-range hopping. Similarly, it is useful to interpret the Wigner ensemble at finite $\alpha$ as an Anderson model under the influence of a constant electric field of strength $\alpha$. This approach enables one to visualize the behavior of the local density of states which is closely related to that of the eigenvectors. In the absence of electric field, the Anderson model is on average translational invariant. Therefore, the ensemble averaged local density of states,

$$
\rho_{L}(E, n) \equiv<\sum_{i}\left|\left(u_{i}\right)_{n}\right|^{2} \delta\left(E-E_{i}\right)>_{e},
$$

where $\left(u_{i}\right)_{n}$ is the $n$-th component of the $i$-th eigenvector of the random matrix and $E_{i}$ the corresponding eigenvalue, is proportional to the average density of states itself, $\rho(E)$. In particular, for the BRME, both $\rho(E)$ and $\rho_{L}(E, n)$ are in the form of a semicircle of radius 
$2 \sqrt{2 b}$. While turning on the electric field breaks the translational invariance, for small enough fields the hopping potential varies much faster than the electric one and the adiabatic approximation that relays on the separation of these two energy scales is known as the sloping band picture. In this regime, one has at each site the same $\rho_{L}(E, n)$ as at zero field only that its center is shifted to include the additional electric energy that is increasing linearly along the lattice. This is schematically illustrated in Fig. 11 where the allowed region inside the band represents the energy shell of the Wigner ensemble. While taking a section through the energy band at a fixed position, $n$, gives $\rho_{L}(E, n)$, a section at a fixed energy is expected to give information on the behavior of the average eigenvector. In particular, one expects that the average eigenvector is constrained to lie within the energy shell. Independently of the sloping band picture, the $\rho_{L}(E, n)$ for the Wigner ensemble was derived in Ref. 8 (see also Ref. 10). It was found that

$$
\rho_{L}(E, n)=\frac{1}{\alpha b} f\left(\frac{E-\alpha n}{\alpha b}, q\right),
$$

where $q \equiv\left(\alpha^{2} b\right)^{-1}$. For small electric fields, $q \gg 1$, the semicircle behavior persists

$$
f(x, q)=(4 \pi q)^{-1} \sqrt{8 q-x^{2}},
$$

and for large fields, $q \ll 1$, the profile is Lorentzian,

$$
f(x, q)=\frac{q}{\pi^{2} q^{2}+x^{2}} .
$$

In fact, Eqs. (8-9) only hold for $x<1$. For $x \gg 1, f$ is the solution of an integral equation for which

$$
f(x, q) \simeq c \exp \left[-2 x \ln \left(x e^{-1} \sqrt{2 q^{-1} \ln (x / \sqrt{q})}\right)\right],
$$

represents an approximate solution.

We now turn to discuss the behavior of the average eigenvector, that is defined as the average variance of the vector component at a fixed distance from the largest component, $\left.g(l) \equiv<\mid\left(u_{i}\right)\left(n-n_{\max }\right)\right)\left.\right|^{2}>_{e}$. We find that the tails of $g(l)$ far outside the energy shell are directly determined by the local density of states, $\rho_{L}(E, n)$ (see Fig. 2). On the other hand, inside the energy shell the shape of $g(l)$ is different in the large and small $q$ regimes. At large electric field, $q \ll 1$, the disorder is too weak to localize the eigenvector and accordingly, like in the case of the tails, $g(l)$ is determined by the local density of states. That is, it takes the Lorentzian form of Eq. (8) with $l=E / \alpha$. For weak field however, $q \gg 1$, the band is only slightly sloped and as a consequence, the variation of the local density of states is slower than the scale on which localization due to disorder takes place. Thus, the disorder is dominant and the same exponentially localized shape as in the absence of the electric field is obtained (see Fig. 3). The transition between the two regimes is centered at $q_{c}$ where the hopping range is of the same size as the energy shell width. A rough estimate of $q_{c}$ can be obtained assuming that the width of the energy shell does not change with $\alpha$ staying $4 \sqrt{2 b}$ all the way down to $q_{c}$. While such estimate gives $q_{c} \approx 0.125$, numerically a value of $q_{c} \simeq 0.09$ is obtained. ${ }^{11}$

In order to establish the correspondence between the various regimes of the Wigner ensemble and the structure of Hamiltonian matrices, one needs to use the semiclassical formulas for the parameters of the ensemble. Notice that the variances of the matrix elements in the Wigner ensemble are $O(1)$ unlike those in $H_{n m}$ and therefore, the effective value of the electric field for the Hamiltonian matrix is $\alpha_{e f}=\alpha / \sigma_{O}$. Then, using Eqs. (2 - 5), one obtains that $q=O\left(\hbar^{-2}\right)$ implying that in the semiclassical limit the $H_{n m}$ matrices are in the disorder dominated regime and therefore exponentially localized eigenvectors can be found whenever the system is sufficiently disordered. This is the main result of the paper.

Let us now study the extent to which the Wigner ensemble is a good model for a particular Hamiltonian matrix,${ }^{12}$ namely, that of the Coupled Quartic Oscillators model,

$$
H=\frac{p_{1}^{2}+p_{2}^{2}}{2}+b_{c} q_{1}^{4}+b_{c}^{-1} q_{2}^{4}-a_{k} q_{1}^{2} q_{2}^{2},
$$

at $b_{c}=\pi / 4$ and $a_{k}=1.6$ where the classical dynamics in the Poincare section appears to be fully chaotic to a resolution of about $0.4 \%$ of $\hbar$ which, in turn, was taken to be unity. Moreover, we take $H_{0}$ to be composed of two uncoupled harmonic oscillators with frequencies $w_{1}=$ 4.11 and $w_{2}=1.3$, truncate the resulting $H_{n m}$ matrix to the first $N$ basis states, $N=800$, and average the eigenvectors to obtain the corresponding $g(l)$ function (see Fig. 3). The comparison with the prediction of the Wigner ensemble that has the same average $b$ and $\alpha$ and the same $N$ shows large quantitative disagreement. In particular, the exponentially decaying shape quickly saturates into broad shoulders that end in a fast drop corresponding to the edge of the energy shell. However, the reasons for this discrepancy are not related to the intrinsic differences between evolution type matrices and Hamiltonian ones, namely the behavior of the diagonal matrix elements since these are irrelevant in the large $q$ regime. Instead, it is a consequence of the fact that the $U_{n m}$ matrix of kicked systems is a lot more random, and thus a lot closer to the BRME, than is the $H_{n m}$ matrix of our example to the Wigner ensemble. In the case of the Kicked Rotor for example, 


$$
U_{n m}=\exp \left(-i \hbar T n^{2} / 2\right)(-i)^{m-n} J_{m-n}(k),
$$

where $J$ are the Bessel functions. The exponential factor originates from the kinetic energy term playing here the role of $H_{0}$ and it constitutes an efficient source of randomness. In the $H_{n m}$ matrix on the other hand, the $H_{0}$ term is both additive and not exponentiated and therefore, the mechanism for generating randomness in Eq. (12) is absent here. It is natural to expect that, in order to fully recover the predictions of the Wigner ensemble, one needs to use more complex bases than that of harmonic oscillators as an alternative source of randomness in the matrix elements of $H_{n m} \cdot{ }^{13}$

In summary, although eigenstates of time-independent Hamiltonian systems are indeed localized inside the energy shell, the quantitative behavior is controlled by the degree of correlation between matrix elements. The study of the way in which such correlations are influenced by the choice of basis is an exciting open question to be addressed in future work. In contrast to the case of the eigenvectors, the eigenvalue statistics is greatly influenced by the behavior of the diagonal matrix elements. Specifically, a finite $\alpha$ makes neighboring eigenvalues correspond to eigenvectors that strongly overlap and the ensuing level repulsion leads to a Wigner type spacing distribution. ${ }^{9}$

We would like to thank B. Horovitz, D.M. Leitner and B. Shapiro for useful discussions. This work was supported by the Israel Science Foundation administered by the Israel Academy of Sciences and Humanities.
1. S. Fishman, D.R. Grempel, and R.E. Prange, Phys. Rev. Lett. 49, 509 (1982).

2. M. Feingold, S. Fishman, D.R. Grempel, and R.E. Prange, Phys. Rev. B31, 6852 (1985).

3. O. Bohigas and M.J. Giannoni, in Mathematical and Computational Methods in Nuclear Physics, edited by J.S. Dehesa, J.M.G. Gomez, and A. Polls, Lecture Notes in Physics Vol. 209 (Springer-Verlag, Berlin, 1984).

4. G. Casati, L. Molinari, and F. Izrailev, Phys. Rev. Lett. 64, 1851 (1990).

5. M.V. Berry, in Chaotic Behavior of Deterministic Systems, Vol. 36 of Les Houches Summer School Proceedings, edited by G. Iooss, R.H.G. Helleman, and R. Stora (North-Holland, Amsterdam, 1983).

6. A.I. Shnirelman, Usp. Mat. Nauk. 29/6, 181 (1974); M. Feingold, N. Moiseyev, and A. Peres, Chem. Phys. Lett. 177, 344 (1985).

7. M. Feingold and A. Peres, Phys. Rev. A34, 591 (1986).

8. E.P. Wigner, Ann. Math. 62, 548 (1955); 65203 (1957).

9. M. Feingold, D.M. Leitner, and M. Wilkinson, Phys. Rev. Lett. 66, 986 (1991); J. Phys. A 24, 175 (1991).

10. D.M. Leitner and M. Feingold, J. Phys. A 26, 7367 (1993); G. Casati, B.V. Chirikov, I. Guarneri, and F.M. Izrailev, Phys. Rev. E 48, 1613 (1993); Y.Y Fyodorov, O.A. Chubykalo, F.M. Izrailev, and G. Casati, Phys. Rev. Lett. 76, 1603 (1996).

11. A similar picture was independently suggested by G. Casati, B.V. Chirikov, I. Guarneri, and F.M. Izrailev, preprint (1995).

12. A previous such comparison was done using a model of the Ce atom in V.V. Flambaum, A.A. Gribakina, G.F. Gribakin, and M.G. Kozlov, Phys. Rev. A50, 267 (1994). Their model has 12 degrees of freedom and no clear classical limit. While, the value of $q$ which they find is close to $q_{c}, q \simeq 0.18$, some agreement with the predictions of the corresponding Wigner ensemble is observed.

13. Another difference between the Wigner ensemble and the $H_{n m}$ matrix studied here is that the latter is not semiclassical enough leading to nonnegligible variation of the band width and mean spacing with $E_{0}$. 
Fig. 1. The sloping band picture. The full lines correspond to the energy band edges. The dashed lines indicate fixed $n$ sections which give the local density of states and fixed energy sections which for small $q$ lead to the average eigenvector.

Fig. 2. The numerically obtained $g(l)$ function for the Wigner ensemble with $\alpha=2$ and $b=14(q=0.0179)(\diamond)$ are compared with Eqs. $(9-10)$. Moreover, the $\times$ symbols represent the numerical $\frac{1}{4} g(l / 4)$ function for the Wigner ensemble with $\alpha=1$ and $b=56$ (same $q$ ), verifying the scaling of Eq. (7) in the $n$ direction.

Fig. 3. The average eigenvector for the Coupled Quartic Oscillators (full) when the matrix has effective $\alpha_{e f}=0.013$ and $b=12.9(q=446)$ and is compared with the corresponding result from the Wigner ensemble with the same $\alpha$ and $b=13$ (dashed). The $\times$ symbols correspond to the $g(l)$ of the Wigner ensemble with the same $b$ but $\alpha=0$, indicating that in this regime, $\alpha$ has almost no influence on the shape of the average eigenvector except for the sharp drop at $l \approx \pm 780$ due to the band edge. 


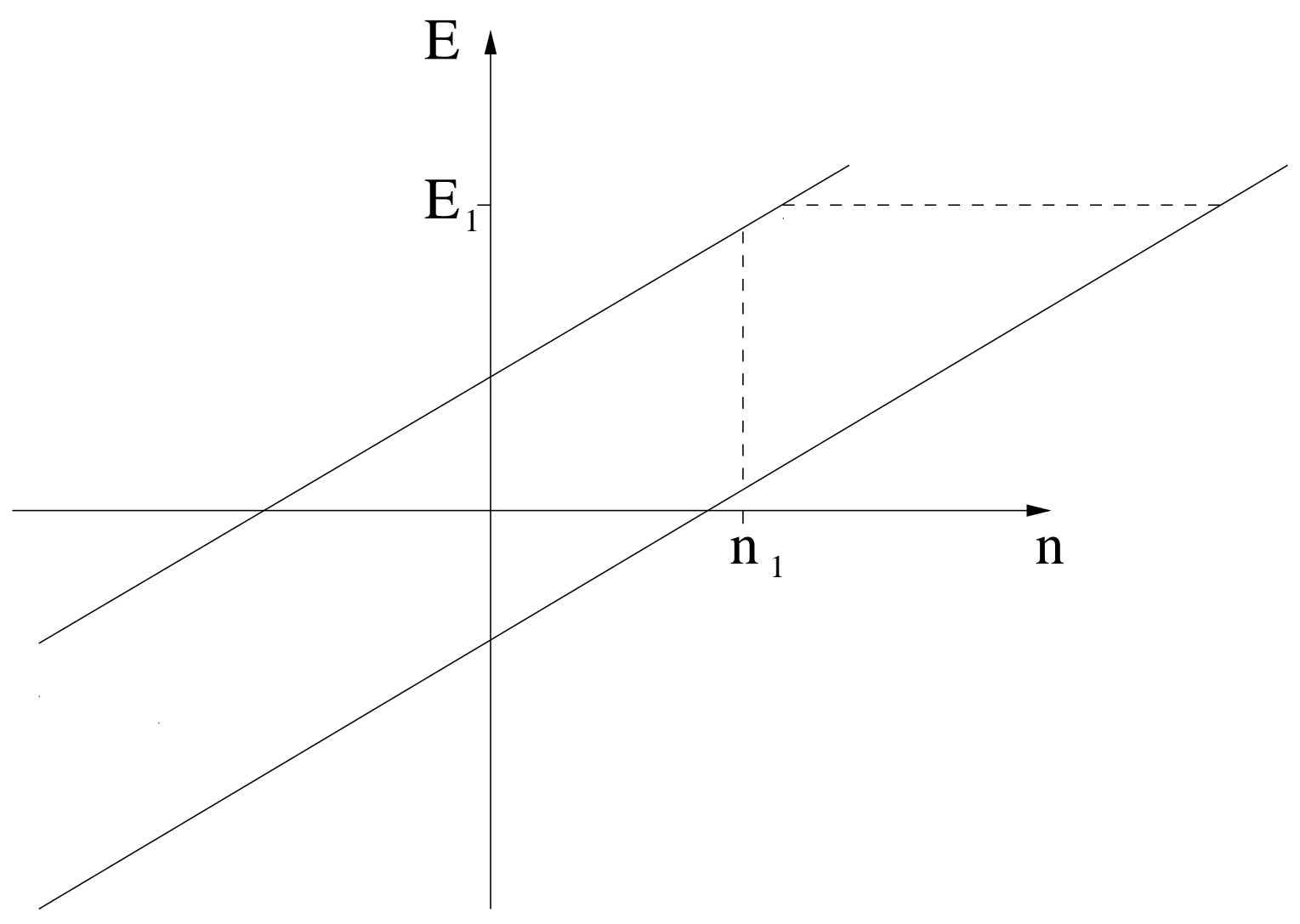




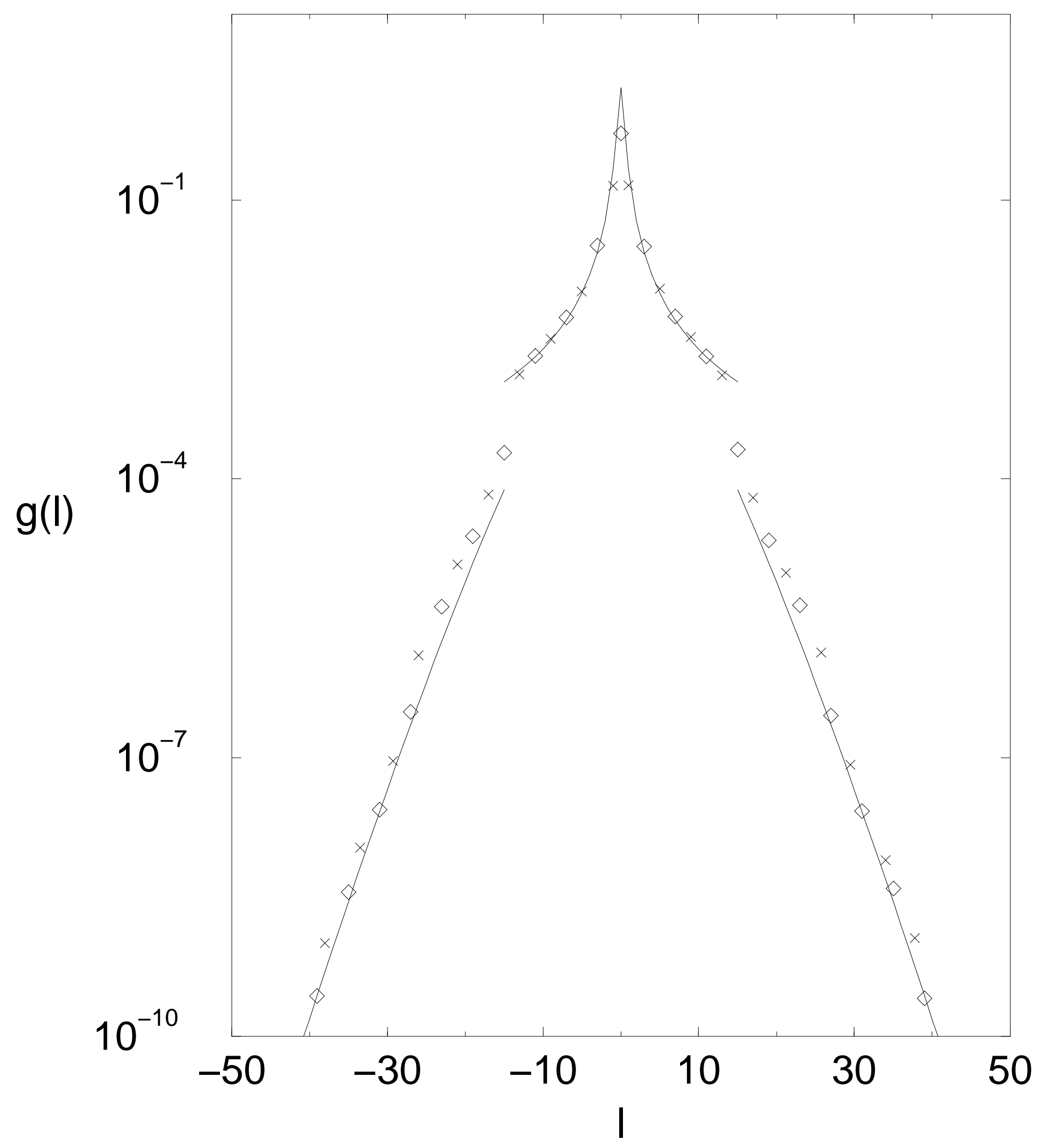




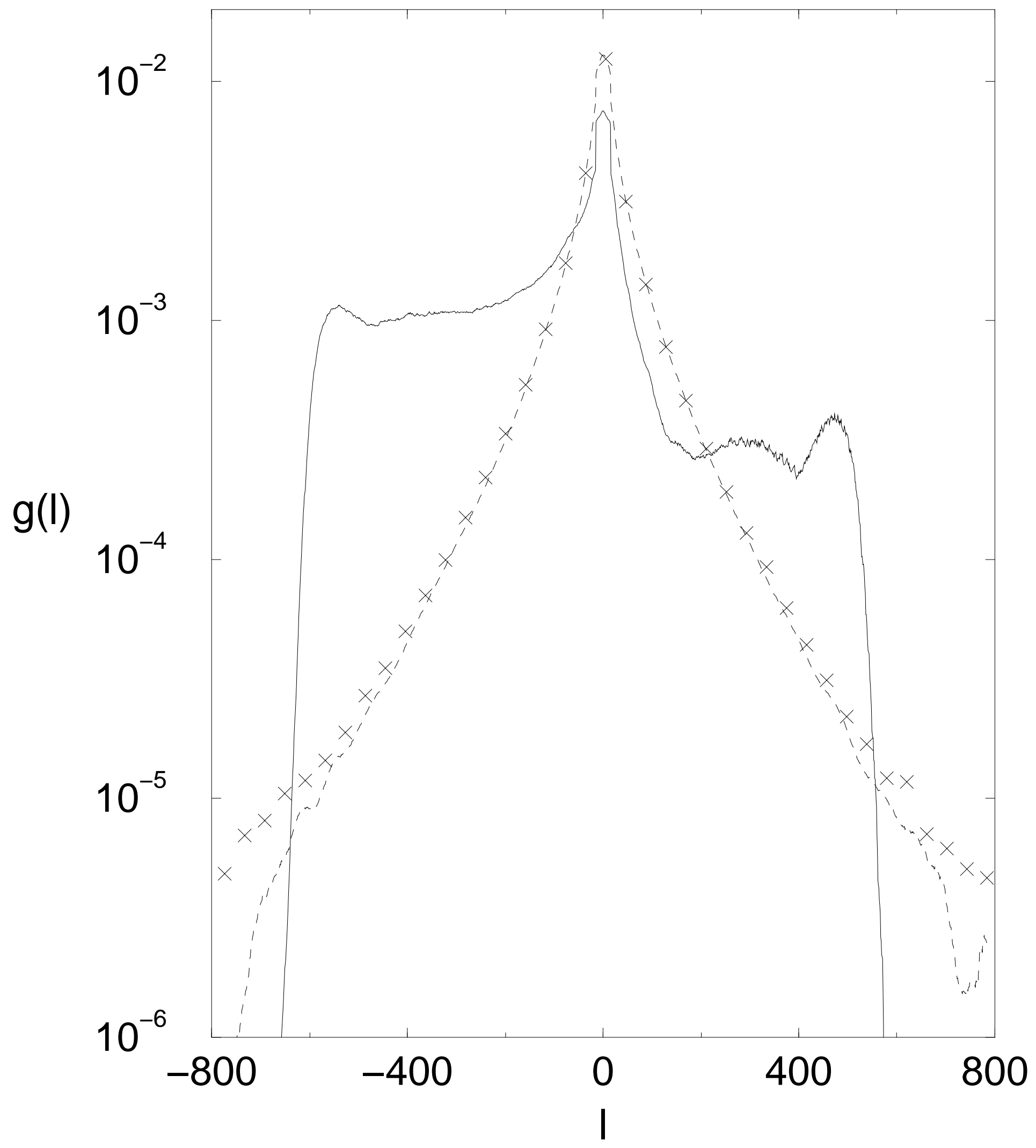

